\title{
SPECIAL FINITE DIFFERENCE METHOD FOR SINGULAR PERTURBATION PROBLEMS WITH ONE-END BOUNDARY LAYER
}

\author{
K. Phaneendra ${ }^{1}$, K. Madhulatha ${ }^{2}$ and Y.N. Reddy ${ }^{2}$ \\ ${ }^{1}$ Department of Mathematics, Nizam College, Osmania University, 500001 \\ Hyderabad, India \\ ${ }^{2}$ Department of Mathematics, National Institute of Technology, Warangal-506004, \\ India \\ kollojuphaneendra@yahoo.co.in,kml.latha3@gmail.com, ynreddy@nitw.ac.in
}

\begin{abstract}
In this paper, we have presented a special finite difference method for solving a singular perturbation problem with layer behaviour at one end. In this method, we have used a second order finite difference approximation for the second derivative, a modified second order upwind finite difference approximation for the first derivative and a second order average difference approximation for $y$ to reduce the global error and retaining tridiagonal system. Then the discrete invariant imbedding algorithm is used to solve the tridiagonal system. This method controls the rapid changes that occur in the boundary layer region and it gives good results in both cases i.e., $h \leq \varepsilon$ and $\varepsilon<<h$. The existence and uniqueness of the discrete problem along with stability estimates are discussed. Also we have discussed the convergence of the method. We have presented maximum absolute errors for the standard examples chosen from the literature.
\end{abstract}

Key Words - Singularly perturbed boundary value problems, Boundary layer, Tridiagonal matrix, diagonally dominant, maximum absolute error.

\section{INTRODUCTION}

Singularly perturbed boundary value problems arise frequently in many areas of science and engineering such as heat transfer problem with large Peclet numbers, Navier-Stokes flows with large Reynolds numbers, chemical reactor theory, aerodynamics, reaction-diffusion process etc. due to the variation in the width of the layer with respect to the small perturbation parameter $\varepsilon$. Several difficulties are experienced in solving the singular perturbation problems using standard numerical methods. Equations of this type typically exhibit solutions with layers; that is, the domain of the differential equation contains narrow regions where the solution derivatives are extremely large.

The numerical treatment of singularly perturbed differential equations gives major computational difficulties due to the presence of boundary and/or interior layers. This type of problem was solved asymptotically by Bellman [1], Bender and Orszag [2], Kevorkian and Cole [3], Nayfeh [4], O'Malley [5] and numerically by Kreiss [6], Miller [7], Kadalbajoo and Devendra Kumar [8], Reddy [9, 10], Lin and Vancouver [11], and Van Veldhuizen [12] etc. It is well-known that replacing the first derivative by central difference is not suitable, i.e., no resemblance at all exists between the solution of the differential equation and the solution of the difference equation. This difficulty can be 
removed by approximating the first derivative by second order modified upwind difference.

\section{DESCRIPTION OF THE METHOD}

\subsection{Left-end boundary layer problems}

To describe this method, we consider a linearly singularly perturbed two point boundary value problem of the form:

$$
\varepsilon y^{\prime \prime}(x)+a(x) y^{\prime}(x)+b(x) y(x)=f(x), \quad x \in[0,1]
$$

with the boundary conditions $y(0)=\alpha$

$$
\text { and } y(1)=\beta
$$

where $\varepsilon$ is a small positive parameter $(0<\varepsilon<<1)$ and $\alpha, \beta$ are known constants. We assume that $a(x), b(x)$ and $f(x)$ are sufficiently continuously differentiable functions in $[0,1]$. Further more, we assume that $b(x) \leq 0, a(x) \geq \mathrm{M}>0$ throughout the interval $[0,1]$, where $M$ is some positive constant. Under these assumptions, (1) has a unique solution $y(x)$ which in general, displays a boundary layer of width $\mathrm{O}(\varepsilon)$ at $x=0$ for small values of $\mathcal{E}$.

Divide the interval $[0,1]$ into $N$ equal parts with constant mesh length $\mathrm{h}$. Let $0=x_{1}, x_{2}, \ldots \ldots x_{N}=1$ be the mesh points. Then we have $x_{i}=i h: i=0,1,2, \ldots, N$.

At $x=x_{i}$ the equation (1) becomes $\varepsilon y_{i}^{\prime \prime}(x)+a_{i} y_{i}^{\prime}+b_{i} y_{i}=f_{i}$

We extend the idea given by Van Veldhuizen [12] to the boundary value problem (1) by considering the special finite difference scheme for the equation (1) as follows:

$$
\begin{gathered}
\varepsilon\left(\frac{y_{i-1}-2 y_{i}+y_{i+1}}{h^{2}}\right)+a_{i}\left(\frac{y_{i+1}-y_{i}}{h}-\frac{h}{2} y_{i}^{\prime \prime}\right)+b_{i}\left(\frac{y_{i+1}+y_{i-1}}{2}\right)=f_{i} \\
\varepsilon\left(\frac{y_{i-1}-2 y_{i}+y_{i+1}}{h^{2}}\right)+a_{i}\left(\frac{y_{i+1}-y_{i}}{h}-\frac{h}{2}\left(\frac{f_{i}-a_{i} y_{i}^{\prime}-b_{i} y_{i}}{\varepsilon}\right)\right)+b_{i}\left(\frac{y_{i+1}+y_{i-1}}{2}\right)=f_{i} \\
\varepsilon\left(\frac{y_{i-1}-2 y_{i}+y_{i+1}}{h^{2}}\right)+a_{i}\left(\frac{y_{i+1}-y_{i}}{h}\right)+\frac{a_{i} a_{i+1 / 2} h}{2 \varepsilon} y_{i}^{\prime}+\frac{a_{i} b_{i+1 / 2} h}{2 \varepsilon} y_{i} \\
+b_{i}\left(\frac{y_{i+1}+y_{i-1}}{2}\right)=f_{i}+\frac{a_{i} h}{2 \varepsilon} f_{i+1 / 2}
\end{gathered}
$$

Substituting $y_{i}^{\prime}=\frac{y_{i+1}-y_{i}}{h}$ in the above equation and simplifying, we get

$$
\begin{aligned}
& \left(\frac{\varepsilon}{h^{2}}+\frac{b_{i}}{2}\right) y_{i-1}-\left(\frac{2 \varepsilon}{h^{2}}+\frac{a_{i}}{h}+\frac{a_{i} a_{i+1 / 2}}{2 \varepsilon}-\frac{a_{i} b_{i+1 / 2} h}{2 \varepsilon}\right) y_{i}+ \\
& \left(\frac{\varepsilon}{h^{2}}+\frac{a_{i}}{h}+\frac{a_{i} a_{i+1 / 2}}{2 \varepsilon}+\frac{b_{i}}{2}\right) y_{i+1}=f_{i}+\frac{a_{i} h}{2 \varepsilon} f_{i+1 / 2}
\end{aligned}
$$

The tridiagonal system of the above equation is:

$E_{i} y_{i-1}-F_{i} y_{i}+G_{i} y_{i+1}=H_{i} ; \quad \mathrm{i}=1,2, \ldots . ., N-1$.

where 


$$
\begin{aligned}
& E_{j}=\frac{\varepsilon}{h^{2}}+\frac{b_{i}}{2}, \quad F_{j}=\frac{2 \varepsilon}{h^{2}}+\frac{a_{i}}{h}+\frac{a_{i} a_{i+1 / 2}}{2 \varepsilon}-\frac{a_{i} b_{i+1 / 2} h}{2 \varepsilon} \\
& G_{j}=\frac{\varepsilon}{h^{2}}+\frac{a_{i}}{h}+\frac{a_{i} a_{i+1 / 2}}{2 \varepsilon}+\frac{b_{i}}{2}, \quad H_{j}=f_{i}+\frac{a_{i} h}{2 \varepsilon} f_{i+1 / 2}
\end{aligned}
$$

We solve the tridiagonal system (6) by using the discrete invariant imbedding algorithm.

\subsection{Right - End Boundary value problems}

Now we assume that $a(x), b(x)$ and $f(x)$ are sufficiently continuously differentiable functions in $[0,1]$ and $b(x) \leq 0, a(x) \leq \mathrm{M}<0$ throughout the interval $[0,1]$, where $M$ is some negative constant. Under these assumptions, (1) has a unique solution $y(x)$, displays a boundary layer of width $\mathrm{O}(\varepsilon)$ at $x=1$ for small values of $\varepsilon$. For the right-end boundary layer problems, we consider the special finite difference scheme as:

$$
\begin{aligned}
& \varepsilon\left(\frac{y_{i-1}-2 y_{i}+y_{i+1}}{h^{2}}\right)+a_{i}\left(\frac{y_{i}-y_{i-1}}{h}+\frac{h}{2} y_{i}^{\prime \prime}\right)+b_{i}\left(\frac{y_{i+1}+y_{i-1}}{2}\right)=f_{i} \\
& \oint\left(\frac{y_{i-1}-2 y_{i}+y_{i+1}}{h^{2}}\right)+a_{i}\left(\frac{y_{i}-y_{i-1}}{h}+\frac{h}{2}\left(\frac{f_{i}-a_{i} y_{i}^{\prime}-b_{i} y_{i}}{\varepsilon}\right)\right)+b_{i}\left(\frac{y_{i+1}+y_{i-1}}{2}\right)=f_{i} \\
& \varepsilon\left(\frac{y_{i-1}-2 y_{i}+y_{i+1}}{h^{2}}\right)+a_{i}\left(\frac{y_{i}-y_{i-1}}{h}\right)-\frac{a_{i} a_{i-1 / 2} h}{2 \varepsilon} y_{i}^{\prime}-\frac{a_{i} b_{i-1 / 2} h}{2 \varepsilon} y_{i}+ \\
& b_{i}\left(\frac{y_{i+1}+y_{i-1}}{2}\right)=f_{i}-\frac{a_{i} h}{2 \varepsilon} f_{i-1 / 2} \\
& \left(\frac{\varepsilon}{h^{2}}-\frac{a_{i}}{h}+\frac{a_{i} a_{i-1 / 2}}{2 \varepsilon}+\frac{b_{i}}{2}-\frac{b_{i} a_{i-1 / 2} h}{2 \varepsilon}\right) y_{i-1}-\left(\frac{2 \varepsilon}{h^{2}}-\frac{a_{i}}{h}+\frac{a_{i} a_{i-1 / 2}}{2 \varepsilon}+\frac{a_{i} b_{i-1 / 2} h}{2 \varepsilon}\right) y_{i} \\
& +\left(\frac{\varepsilon}{h^{2}}+\frac{b_{i}}{2}\right) y_{i+1}=f_{i}-\frac{a_{i} h}{2 \varepsilon} f_{i-1 / 2}
\end{aligned}
$$

Equation (9) can be written as the tridiagonal system of the form:

$$
E_{i} y_{i-1}-F_{i} y_{i}+G_{i} y_{i+1}=H_{i} ; \quad \mathrm{i}=1,2, \ldots . ., N-1 \text {. }
$$

where

$$
\begin{aligned}
& E_{j}=\frac{\varepsilon}{h^{2}}-\frac{a_{i}}{h}+\frac{a_{i} a_{i-1 / 2}}{2 \varepsilon}+\frac{b_{i}}{2}-\frac{b_{i} a_{i-1 / 2} h}{2 \varepsilon}, F_{j}=\frac{2 \varepsilon}{h^{2}}-\frac{a_{i}}{h}+\frac{a_{i} a_{i-1 / 2}}{2 \varepsilon}+\frac{a_{i} b_{i-1 / 2} h}{2 \varepsilon} \\
& G_{j}=\frac{\varepsilon}{h^{2}}+\frac{b_{i}}{2}, H_{j}=f_{i}-\frac{a_{i} h}{2 \varepsilon} f_{i-1 / 2}
\end{aligned}
$$

We solve the tridiagonal system (10) by using the discrete invariant imbedding algorithm. Since Eq. (6) or (10) holds for $\mathrm{i}=1,2, \ldots, N-1$, we have $N-1$ linear equations in the $N$-1 unknowns $y_{1}, y_{2}, \ldots . y_{N-1}$. 


\section{STABILITY AND CONVERGENCE ANALYSIS}

Theorem 1. Under the assumptions $\varepsilon>0, a(x) \geq M>0$ and $b(x)<0, \forall x \in[0,1]$, the solution to the system of the difference equations (6), together with the given boundary conditions exists, is unique and satisfies

$$
\|y\|_{h, \infty} \leq M^{-1}\|f\|_{h, \infty}+(|\alpha|+|\beta|)
$$

where \|\|$_{h, \infty}$ is the discrete $l_{\infty}-$ norm, given by $\|x\|_{h, \infty}=\max _{0 \leq \mathrm{i} \leq \mathrm{N}}\left\{\left|x_{i}\right|\right\}$.

Proof. Let $L_{h}$ (.) denote the difference operator on left hand side of Eq. (6) and $w_{i}$ be any mesh function satisfying $L_{h}\left(w_{i}\right)=f_{i}$

By rearranging the difference scheme (6) and using non-negativity of the coefficients $E_{i}, F_{i}$ and $G_{i}$, we obtain $F_{i}\left|w_{i}\right| \leq\left|H_{i}\right|+E_{i}\left|w_{i-1}\right|+G_{i}\left|w_{i+1}\right|$

Now using the assumption $\varepsilon>0$ and $a_{i} \geq M$, the definition of $l_{\infty}$-norm and manipulating the above inequality, we obtain

$$
\begin{aligned}
& \varepsilon \frac{\left(\left|w_{i+1}\right|-2\left|w_{i}\right|+\left|w_{i-1}\right|\right)}{h^{2}}+a_{i}\left(\frac{\left|w_{i+1}\right|-\left|w_{i}\right|}{2 h}\right)+\frac{a_{i} a_{i+1 / 2}}{2 \varepsilon}\left(\left|w_{i+1}\right|-\left|w_{i}\right|\right) \\
& +\frac{b_{i}}{2}\left(\left|w_{i-1}\right|+\left|w_{i+1}\right|\right)+\frac{a_{i} b_{i+1 / 2} h}{2 \varepsilon}\left|w_{i}\right|+\left|f_{i}\right|+\frac{a_{i} h}{2 \varepsilon}\left|f_{i+1 / 2}\right| \geq 0
\end{aligned}
$$

To prove the uniqueness and existence, let $\left\{u_{i}\right\},\left\{v_{i}\right\}$ be two sets of solution of the difference equation (6) satisfying boundary conditions. Then $w_{i}=u_{i}-v_{i}$

satisfies $L_{h}\left(w_{i}\right)=f_{i}$ where $f_{i}=0$ and $w_{0}=w_{N}=0$.

Summing (11) over $i=1,2, \ldots \ldots, N-1$, we obtain

$$
-\varepsilon \frac{\left|w_{1}\right|}{h^{2}}-\varepsilon \frac{\left|w_{N-1}\right|}{h^{2}}-\left(\frac{\|a\|_{h, \infty}}{h}+\frac{\|a\|_{h, \infty}^{2}}{2 \varepsilon}\right)\left\|w_{1}\right\|+\frac{b_{i}}{2} \sum_{i=1}^{N-2}\left|w_{i}\right|+\frac{\|a\| b h}{2 \varepsilon} \sum_{i=1}^{N-1}\left|w_{i}\right| \geq 0
$$

Since $\quad \varepsilon>0,\|a\|_{h, \infty} \geq 0, b_{i}<0$ and $\left|w_{i}\right| \geq 0 \forall i, i=1,2, \ldots \ldots, N-1, \quad$ therefore $\quad$ for inequality (12) to hold, we must have $w_{i}=0 \quad \forall i, i=1,2, \ldots . . N-1$.

This implies the uniqueness of the solution of the tridiagonal system of difference equations (6). For linear equations, the existence is implied by uniqueness. Now to establish the estimate, let $w_{i}=y_{i}-l_{i}$, where $y_{i}$ satisfies difference equations (6), the boundary conditions and $l_{i}=(1-i h) \alpha+(i h) \beta, \quad$ then $\quad w_{0}=w_{N}=0, \quad$ and $w_{i}, i=1,2, \ldots N-1$.

Now let $\left|w_{n}\right|=\|w\|_{h, \infty} \geq\left|w_{i}\right|, i=0,1, \ldots \ldots ., N$.

Then summing (11) from $i=n$ to $N-1$ and using the assumption on $a(x)$, which gives 
$-\varepsilon \frac{\left(\left|w_{n}\right|-\left|w_{n-1}\right|\right)}{h^{2}}-\varepsilon \frac{\left|w_{N-1}\right|}{h^{2}}-\frac{M}{h}\left|w_{n}\right|-\frac{h M^{2}}{2 \varepsilon}\left|w_{n}\right|+\frac{1}{2} \sum_{i=n-1}^{N-2} b_{i}\left|w_{i}\right|+\frac{M h}{2 \varepsilon} \sum_{i=n}^{N-1} b_{i+1 / 2}\left|w_{i}\right|$

$\frac{M h}{2 \varepsilon} \sum_{i=n}^{N-1}\left|f_{i+1 / 2}\right|+\sum_{i=n}^{N-1}\left|f_{i}\right| \geq 0$

Inequality (13), together with the condition on $b(x)$ implies that

$M\left|w_{n}\right| \leq h \sum_{i=n}^{N-1}\left|f_{i}\right| \leq h \sum_{i=0}^{N}\left|f_{i}\right| \leq\|f\|_{h, \infty}$,

i.e., we have

$$
\left|w_{n}\right| \leq M^{-1}\|f\|_{h, \infty}
$$

Also, we have $y_{i}=w_{i}+l_{i}$,

$$
\|y\|_{h, \infty}=\max _{0 \leq i \leq N}\left\{\left|y_{i}\right|\right\} \leq\|w\|_{h, \infty}+\|l\|_{h, \infty} \leq\left|w_{h}\right|+\|l\|_{h, \infty} .
$$

Now to complete the estimate, we have to find out the bound on $l_{i}$

$\|l\|_{h, \infty}=\max _{0 \leq i \leq N}\left\{\left|l_{i}\right|\right\} \leq \max _{0 \leq i \leq N}\{|(1-i h)||\alpha|+|i h||\beta|\} \leq \max _{0 \leq i \leq N}\{(1-i h)|\alpha|+(i h)|\beta|\}$,

i.e., we have $\|l\|_{h, \infty} \leq|\alpha|+|\beta|$.

From Eqs. (14) - (15), we obtain the estimate $\|y\|_{h, \infty} \leq M^{-1}\|f\|_{h, \infty}+(|\alpha|+|\beta|)$.

This theorem implies that the solution to the system of the difference equations (6) is uniformly bounded. Thus the scheme is stable for all step sizes.

Corollary 1. Under the conditions for theorem 1, the error $e_{i}=y\left(x_{i}\right)-y_{i}$ between the solution $y(x)$ of the continues problem and the solution $y_{i}$ of the discretized problem, with boundary conditions, satisfies the estimate

$\|e\|_{h, \infty} \leq M^{-1}\|\tau\|_{h, \infty}$, where

$$
\begin{aligned}
\left|\tau_{i}\right| \leq \max _{x_{i-1} \leq x \leq x_{i+1}}\left\{\frac{h^{2}}{2} b(x)\left|y^{\prime \prime}(x)\right|\right\}+\max _{x_{i-1} \leq x \leq x_{i+1}}\left\{\frac{h^{2}}{6} a(x)\left|y^{\prime \prime \prime}(x)\right|\right\} \\
+\max _{x_{i-1} \leq x \leq x_{i+1}}\left\{\frac{h^{2}}{24}\left(1+h a(x)+h^{2} b(x)\right)\left|y^{(4)}(x)\right|\right\}
\end{aligned}
$$

Proof. Truncation error $\tau_{i}$ is given by

$$
\begin{aligned}
& \tau_{i}= \varepsilon\left\{\left(\frac{y_{i+1}-y_{i}+y_{i-1}}{h^{2}}\right)-y_{i}^{\prime \prime}\right\}+a(x)\left\{\left(\frac{y_{i+1}-y_{i-1}}{2 h}\right)-\frac{h}{2} y_{i}^{\prime \prime}-y_{i}^{\prime}\right\}+b(x)\left\{\left(\frac{y_{i+1}+y_{i-1}}{2}\right)-y_{i}\right\} \\
&\left|\tau_{i}\right| \leq \max _{x_{i-1} \leq x \leq x_{i+1}}\left\{\frac{h^{2} b(x)}{2}\left|y^{\prime \prime}(x)\right|\right\}+\max _{x_{i-1} \leq x \leq x_{i+1}}\left\{\frac{h^{2} a(x)}{6}\left|y^{\prime \prime \prime}(x)\right|\right\} \\
&+\max _{x_{i-1} \leq x \leq x_{i+1}}\left\{\frac{\varepsilon h^{2}}{24}\left(1+h a(x)+h^{2} b(x)\right)\left|y^{(4)}(x)\right|\right\}
\end{aligned}
$$

One can easily show that the error $e_{i}$, satisfies 
$L_{h}\left(e\left(x_{i}\right)\right)=L_{h}\left(y\left(x_{i}\right)\right)-L_{h}\left(y_{i}\right)=\tau_{i}, i=1,2, \ldots, N-1$ and $e_{0}=e_{N}=0$.

Then Theorem 1 implies that $\|e\|_{h, \infty} \leq M^{-1}\|\tau\|_{h, \infty}$.

The estimate (17) establishes the convergence of the difference scheme for the fixed values of the parameter $\varepsilon$.

Theorem 2. Under the assumptions $\varepsilon>0, a(x) \leq M<0$ and $b(x)<0, \forall x \in[0,1]$ the solution to the system of the difference equations (10), together with the given boundary conditions exists, is unique and satisfies

$$
\|y\|_{h, \infty} \leq M^{-1}\|f\|_{h, \infty}+(|\alpha|+|\beta|) .
$$

The proof of this estimate can be done on similar lines as we did in theorem 1.

\section{NUMERICAL EXAMPLES}

To demonstrate the applicability of the method we have applied it on linear, nonlinear singular perturbation problems with left-end and right-end boundary layer. The numerical solutions are compared with the exact solutions and maximum absolute errors are presented to support the given method.

Example 1. Consider the following homogeneous singular perturbation problem from Bender and Orszag [2] $\varepsilon y^{\prime \prime}(x)+y^{\prime}(x)-y(x)=0 ; x \in[0,1]$ with $y(0)=1$ and $y(1)=1$.

Clearly this problem has a boundary layer at $x=0$.

The exact solution is given by

$$
y(x)=\frac{\left[\left(e^{m_{2}}-1\right) e^{m_{1} x}+\left(1-e^{m_{1}}\right) e^{m_{2} x}\right]}{\left[e^{m_{2}}-e^{m_{1}}\right]}
$$

where $m_{1}=(-1+\sqrt{1+4 \varepsilon}) /(2 \varepsilon)$ and $m_{2}=(-1-\sqrt{1+4 \varepsilon}) /(2 \varepsilon)$

The maximum absolute errors are presented in tables 1 and 2 for different values of $\varepsilon$.

Example 2. Consider the following variable coefficient singular perturbation problem $\varepsilon y^{\prime \prime}(x)+\left(1-\frac{x}{2}\right) y^{\prime}(x)-\frac{1}{2} y(x)=0 ; x \in[0,1]$ with $y(0)=0$ and $y(1)=1$.

We have chosen to use uniformly valid approximation (which is obtained by the method given by Nayfeh [4] as our 'exact' solution:

$$
y(x)=\frac{1}{2-x}-\frac{1}{2} e^{-\left(x-x^{2} / 4\right) / \varepsilon}
$$

The maximum absolute errors with fitting factor are presented in tables 4 for different values of $\varepsilon$.

Example 3. Consider the following non linear singular perturbation problem from Bender and Orszag [[2], page 463; equations: 9.7.1]

$\varepsilon y^{\prime \prime}(x)+2 y^{\prime}(x)+e^{y(x)}=0 ; \mathrm{x} \in[0,1]$

with $\mathrm{y}(0)=0$ and $\mathrm{y}(1)=0$.

The linear problem concerned to this example is

$$
\varepsilon y^{\prime \prime}(x)+2 y^{\prime}(x)+\frac{2}{x+1} y(x)=\left(\frac{2}{x+1}\right)\left[\log _{e}\left(\frac{2}{x+1}\right)-1\right]
$$


We have chosen to use Bender and Orszag's uniformly valid approximation [[2], page 463; equation: 9.7.6] for comparison,

$y(x)=\log _{e}\left(\frac{2}{x+1}\right)-\left(\log _{e} 2\right) e^{-2 x / \varepsilon}$

For this example, we have boundary layer of thickness $\mathrm{O}(\varepsilon)$ at $\mathrm{x}=0$.

The maximum absolute errors are presented in table 3 for small values of $\varepsilon$.

Example 4. Consider the following singular perturbation problem

$\varepsilon y^{\prime \prime}(x)-y^{\prime}(x)=0 ; x \in[0,1]$ with $y(0)=1$ and $y(1)=0$.

Clearly, this problem has a boundary layer at $\mathrm{x}=1$. i.e., at the right end of the underlying interval.

The exact solution is given by $y(x)=\frac{\left(e^{(x-1) / \varepsilon}-1\right)}{\left(e^{-1 / \varepsilon}-1\right)}$

The maximum absolute errors are presented in tables 5 for different values of $\varepsilon$ and the maximum absolute errors with classical difference scheme are presented in table 6 for comparison.

Example 5. Now we consider the following singular perturbation problem $\varepsilon y^{\prime \prime}(x)-y^{\prime}(x)-(1+\varepsilon) y(x)=0 ; x \in[0,1]$

with $y(0)=1+\exp (-(1+\varepsilon) / \varepsilon)$; and $y(1)=1+1 / \mathrm{e}$.

The exact solution is given by $y(\mathrm{x})=\mathrm{e}^{(1+\varepsilon)(\mathrm{x}-1) / \varepsilon}+\mathrm{e}^{-\mathrm{x}}$

The maximum absolute errors are presented in tables 7 for different values of $\varepsilon$.

\section{DISCUSSIONS AND CONCLUSIONS}

We have described a special finite difference method for solving a singular perturbation problem with layer behaviour at on end point. In this method, we have used a second order finite difference approximation for second order derivative, a modified second order upwind finite difference approximation for first order derivative and a second order average difference approximation for $y$. This method controls the rapid changes that occur in the boundary layer region and it gives good results in the case when $\varepsilon<<h$. We have presented maximum absolute errors for the standard examples chosen from the literature with the proposed method and compared with the classical second order finite difference method to show the efficiency of the method when $\varepsilon<<h$. The computational rate of convergence is also obtained by using the double mesh principle defined below.

Let $Z_{h}=\max _{j}\left|y_{j}^{h}-y_{j}^{h / 2}\right|, j=0,1,2, \ldots, N-1$ where $y_{j}^{h}$ is the computed solution on the mesh $\left\{x_{j}\right\}_{0}^{N}$ at the nodal point $x_{j}$ where $x_{j}=x_{j-1}+h, j=1,2, \ldots . . N$ and $y_{j}^{h / 2}$ is the computed solution at the nodal point $x_{j}$ on the mesh $\left\{x_{j}\right\}_{0}^{2 N}$ where $x_{j}=x_{j-1}+h / 2, j=1(1) 2 N$. In the same way we can define $Z_{h / 2}$ by replacing $h$ by $h / 2$ and $N$ by $2 N$ i.e., $Z_{h / 2}=\max _{j}\left|y_{j}^{h / 2}-y_{j}^{h / 4}\right|, j=0,1,2, . ., 2 N-1$. 
The computed order of convergence is defined as Order $=\frac{\log Z_{h}-\log Z_{h / 2}}{\log (2)}$

We have taken $h=2^{-3}$ for finding the computed order of convergence and results are shown in Table 8.

Table 1. The maximum absolute errors in solution of example 1

\begin{tabular}{|c|c|c|c|c|c|c|c|c|}
\hline \multirow{2}{*}{$\varepsilon \backslash h$} & \multicolumn{2}{|c|}{$2^{-3}$} & \multicolumn{2}{|c|}{$2^{-4}$} & \multicolumn{2}{|c|}{$2^{-5}$} & \multicolumn{2}{|c|}{$2^{-6}$} \\
\hline & $\begin{array}{l}\text { Present } \\
\text { method }\end{array}$ & $\begin{array}{c}\text { Class. } 2^{\text {nd }} \text { order } \\
\text { method }\end{array}$ & $\begin{array}{l}\text { Present } \\
\text { method }\end{array}$ & $\begin{array}{l}\text { Class. } 2^{\text {nd }} \text { order } \\
\text { method }\end{array}$ & $\begin{array}{l}\text { Present } \\
\text { method }\end{array}$ & $\begin{array}{l}\text { Class. } 2^{\text {nd }} \text { order } \\
\text { method }\end{array}$ & & $\begin{array}{l}\text { Class. } 2^{\text {nd }} \text { order } \\
\text { method }\end{array}$ \\
\hline-10 & $2.04(-2)$ & $6.87(-1)$ & $6(-2)$ & 6.21( & $5.30(-3)$ & 5. & $2.50(-3)$ & $4.83(-1)$ \\
\hline $2^{-12}$ & $2.14(-2)$ & 7.3 & $1.10(-2)$ & $7.32(-1)$ & $5.60(-3)$ & 6.58 & $2.90(-3)$ & $5.87(-1)$ \\
\hline $2^{-15}$ & $2.16(-2)$ & $7.54(-1)$ & $1.11(-2)$ & $7.83(-1)$ & $5.60(-3)$ & $7.81(-1)$ & $2.80(-3)$ & $7.30(-1)$ \\
\hline $2^{-20}$ & $2.16(-2)$ & $7.56(-1)$ & $1.12(-2)$ & $7.91(-1)$ & $5.70(-3)$ & $8.09(-1)$ & $2.90(-3)$ & $8.16(-1)$ \\
\hline
\end{tabular}

Table 2. The maximum absolute errors in solution of example 2

\begin{tabular}{|c|c|c|c|c|c|c|c|c|}
\hline \multirow{2}{*}{$\varepsilon \backslash h$} & \multicolumn{2}{|c|}{$2^{-3}$} & \multicolumn{2}{|l|}{$2^{-4}$} & \multicolumn{2}{|l|}{$2^{-5}$} & \multicolumn{2}{|c|}{$2^{-6}$} \\
\hline & $\begin{array}{c}\text { Present } \\
\text { method }\end{array}$ & metnod & $\begin{array}{l}\text { Present } \\
\text { method }\end{array}$ & $\begin{array}{l}\text { Class. } 2^{\text {nd }} \text { order } \\
\text { ethod }\end{array}$ & $\begin{array}{l}\text { Present C } \\
\text { method }\end{array}$ & & & \\
\hline 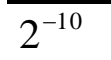 & $2.93(-2)$ & $5(-1)$ & $1.51(-2)$ & $5.22(-1)$ & $7.70(-3)$ & 4.3 & $4.00(-3)$ & 3. \\
\hline $2^{-12}$ & $2.92(-2)$ & 1) & $1.52(-2)$ & 1) & $7.70(-3)$ & & $(-3)$ & $4.67(-1)$ \\
\hline $2^{-15}$ & $2.92(-2)$ & ) & 1.51 & 8. & 7.70 & 1) & $-3)$ & $(-1)$ \\
\hline $0-20$ & $2.92(-2)$ & $8.12(-1)$ & $1.51(-2)$ & $8.19(-1)$ & $7.70(-3)$ & 8.2 & $3.90(-3)$ & $8.20(-1)$ \\
\hline
\end{tabular}

Table 3. The maximum absolute errors in the solution of example 3

\begin{tabular}{|c|c|c|c|c|c|c|c|c|}
\hline \multirow{2}{*}{$\varepsilon \backslash h$} & \multicolumn{2}{|c|}{$2^{-3}$} & \multicolumn{2}{|c|}{$2^{-4}$} & \multicolumn{2}{|c|}{$2^{-5}$} & \multicolumn{2}{|c|}{$\overline{2^{-6}}$} \\
\hline & $\begin{array}{l}\text { Present } \\
\text { method }\end{array}$ & $\begin{array}{c}\text { Class. } \\
\text { metl }\end{array}$ & method & me & & $\begin{array}{r}\text { Class. } \\
\text { met }\end{array}$ & & \\
\hline & 4.4 & & & & $(-2)$ & & & \\
\hline-12 & & & & & $(-2)$ & & & \\
\hline $2^{-15}$ & $4.53(-2)$ & & 2) & & $(-2)$ & & (-J) & 1.336 \\
\hline-20 & $4.54(-2)$ & 1.0841 & $2.57(-2)$ & 1.1102 & $1.37(-2)$ & 1.1358 & $7.10(-3)$ & 1.1495 \\
\hline
\end{tabular}

Table 4. The maximum absolute errors in the solution of example 4

\begin{tabular}{|c|c|c|c|c|c|c|c|c|}
\hline \multirow{2}{*}{$\varepsilon \backslash h$} & \multicolumn{2}{|c|}{$2^{-3}$} & \multicolumn{2}{|l|}{$2^{-4}$} & \multicolumn{2}{|l|}{$2^{-5}$} & \multicolumn{2}{|c|}{$2^{-6}$} \\
\hline & & & & & & & & \\
\hline & & & & & & & & \\
\hline & & & & & & & & \\
\hline & $1.19(-7)$ & 59 & $(-7)$ & & $(-6)$ & & $7.59(-6)$ & \\
\hline & $.16(-10)$ & ) 8. & $4.65(-10)$ & & $6(-9)$ & & $7.49(-9)$ & 127 \\
\hline
\end{tabular}


216 Special Finite Difference Method for Singular Perturbation Problems

Table 5. The maximum absolute errors in the solution of example 5

\begin{tabular}{|c|c|c|c|c|c|c|c|c|}
\hline & \multicolumn{2}{|c|}{$\overline{2^{-3}}$} & \multicolumn{2}{|l|}{$2^{-4}$} & \multicolumn{2}{|c|}{$2^{-5}$} & \multicolumn{2}{|c|}{$\overline{2^{-6}}$} \\
\hline & $\begin{array}{l}\text { Present } \\
\text { method }\end{array}$ & $\begin{array}{l}\text { Class. } 2^{\text {nd }} \text { order } \\
\text { method }\end{array}$ & $\begin{array}{l}\text { Present } \\
\text { method }\end{array}$ & $\begin{array}{l}\text { Class. } 2^{\text {nd }} \text { order } \\
\text { method }\end{array}$ & $\begin{array}{l}\text { Present } \\
\text { method }\end{array}$ & $\begin{array}{l}\text { Class. } 2^{\text {nd }} \text { order } \\
\text { method }\end{array}$ & $\begin{array}{l}\text { Present } \\
\text { method }\end{array}$ & $\begin{array}{l}\text { Class. } 2^{\text {nd }} \text { order } \\
\text { method }\end{array}$ \\
\hline $2^{-10}$ & $2.06(-2)$ & 1.0893 & $1.07(-2)$ & 0.9842 & $6.20(-3)$ & 0.8598 & $8.50(-3)$ & 0.7657 \\
\hline $2^{-12}$ & $2.13(-2)$ & 1.1682 & $1.10(-2)$ & 1.1591 & $5.60(-3)$ & 1.0426 & $3.00(-3)$ & 0.9296 \\
\hline $2^{-15}$ & $2.16(-2)$ & 1.1942 & $1.11(-2)$ & 1.2398 & $5.60(-3)$ & 1.2363 & $2.80(-3)$ & 1.1550 \\
\hline $2^{-20}$ & $2.16(-2)$ & 1.1979 & $1.12(-2)$ & 1.2526 & $5.70(-3)$ & 1.2809 & $2.90(-3)$ & 1.2917 \\
\hline
\end{tabular}

Table 6. Numerical order of convergence for examples 1-5

\begin{tabular}{lcccc}
\hline & $h$ & $h / 2$ & $Z_{h}$ & Order of convergence \\
\hline Example 1 & $2^{-3}$ & $2^{-4}$ & $7.90 \mathrm{E}-03$ & \\
& $2^{-4}$ & $2^{-5}$ & $3.00 \mathrm{E}-03$ & 1.3897 \\
Example 2 & $2^{-3}$ & $2^{-4}$ & $5.10 \mathrm{E}-03$ & \\
& $2^{-4}$ & $2^{-5}$ & $3.00 \mathrm{E}-03$ & 0.7573 \\
Example 3 & $2^{-3}$ & $2^{-4}$ & $3.02 \mathrm{E}-02$ & \\
& $2^{-4}$ & $2^{-5}$ & $1.20 \mathrm{E}-02$ & 1.3373 \\
Example 4 & $2^{-3}$ & $2^{-4}$ & $2.12 \mathrm{E}-02$ & 1.4811 \\
& $2^{-4}$ & $2^{-5}$ & $7.60 \mathrm{E}-03$ & 1.3296 \\
\hline
\end{tabular}

\section{REFERENCES}

1. R. Bellman, Perturbation Techniques in Mathematics, Physics and Engineering, Holt, Rinehart \& Winston, New York, 1964.

2. C.M. Bender, S.A. Orszag, Advanced mathematical methods for scientists and engineers, Mc. Graw-Hill, New York, 1978.

3. M.K. Kadalbajoo, Devendra Kumar, Initial value technique for singularly perturbed two point boundary value problems using an exponentially fitted finite difference scheme, Computers and Mathematics with Applications, 57, 1147-1156, 2009.

4. A.H. Nayfeh, Introduction to Perturbation Methods, John Wiley and Sons, New York, 1981.

5. R.E. O’Malley, Introduction to Singular Perturbations, Academic Press, New York, 1974.

6. B. Kreiss, H.O. Kreiss, Numerical methods for singular perturbation problems, SIAM Journal on Numerical Analysis 46, 138-165, 1982.

7. P. Lin, Vancouver, A Numerical solution of quasilinear singularly perturbed ordinary differential equation without turning points, Applied Mathematics and Mechanics 10, 1005-1010, 1989. 
8. J. Kevorkian, J.D. Cole, Multiple Scale and Singular Perturbation Methods, Springer-Verlag, New York, 1996.

9. M.K. Kadalbajoo, Y.N. Reddy, A non asymptotic method for general singular perturbation problems, Journal of Optimization Theory and Applications, 55, 256-269, 1986.

10. Y.N. Reddy, A Numerical integration method for solving singular perturbation problems, Applied Mathematics and Computation 37, 83-95, 1990.

11. J.J.H. Miller, E.O. Riordan, G.I. Shishkin, On piecewise uniform meshes for upwind and central difference operators for solving singularly perturbed problems, IMA Journal of Numerical Analysis 15, 89-99, 1995.

12. M. Van Veldhuizen, Higher order schemes of positive type for singular perturbation problems, in P. W. Hemker, J.J.H. Miller, Numerical analysis of singular perturbation problems, Academic Press, New York, 361-383, 1979. 